\title{
Survey of specialised UK traumatic stress services
}

\section{AIMS AND METHOD}

The aim of the survey was to establish the organisational structure and practice of specialised services for post-traumatic stress in the UK. Questionnaires were collected from 17 specialised trauma services across the UK.

\section{RESULTS}

Specialised trauma services use similar therapeutic programmes, but differ with respect to the characteristics of the treated clientele and organisational features. Although almost all services routinely measure outcome, some of the instruments used vary. There is no clear association between staff resources and number of patients treated.

\section{CLINICAL IMPLICATIONS}

Specialised traumatic stress services in the UK employ evidence-based treatment methods. A uniform protocol to measure outcome may help to establish a common UK-wide database on outcome of specialised treatment, and facilitate a reliable comparison between different service organisations and programmes. Organisational features should be considered to increase the efficiency of services.
People with trauma-related mental disorders are treated within different services in the National Health Service (NHS) in primary, secondary and tertiary care. The provision is still subject to local variation but the National Institute for Clinical Excellence (NICE) has developed guidelines for the management of post-traumatic stress disorder (PTSD) in adults in primary, secondary and community care (National Collaborating Centre for Mental Health, 2005). However, these guidelines focus more on treatment methods than the organisation of specialised services.

The number of specialised trauma services has increased in the last decade. These services are depending on the terminology - located in specialised secondary or tertiary care. Reasons for the increase in their number may include additional funding for mental healthcare in general and a significant influx of refugees into the UK - a population which has had a higher exposure to trauma and has a higher prevalence of PTSD than the UK general population (Berthold, 2000; Howard \& Hodes, 2000; Porter \& Haslam, 2001).

The aim of this survey was to establish: (a) how specialised trauma services in the UK are organised and staffed; (b) what treatment programmes they provide; (c) how many patients they care for; and (d) how they assess treatment outcome. Such information should complement the NICE guidelines as a basis for further service development.

\section{Method}

\section{Measures}

A postal questionnaire was designed specifically for this survey (available from the authors on request). Respondents were asked: to record mental health professionals who work in services; type of referrals; details about the assessment, treatment and support provided; supervision in service; and outcome measures used. Categories of questions and answers are summarised in Box 1.

\section{Data collection}

Specialist trauma services included in the survey were identified through the UK Trauma Group (UKTG) website, which is an informal network of practitioners working with traumatised people in the UK. The UK Trauma Group includes clinicians who run larger, multiprofessional, specialist centres or research teams and who expressed a commitment to evidence-based practice (UK Trauma Group, 2004).

All specialist trauma services found on the website were contacted. Initial contacts were made via post or e-mail, with a brief explanation of the aims of the survey and an invitation to complete a short postal questionnaire. If no responses were received, further contact was made via telephone or personally.

Out of 23 services contacted from the list, five replied that the survey was non-applicable to them (e.g. because they regarded themselves as a secondary mental health service with a special interest in trauma but not as a specific trauma service). One service reported that such information was confidential. Hence, 17 responses were received and included in the analysis.

In terms of statistical methods, descriptive statistics as well as bivariate parametric and non-parametric correlations have been used.

\section{Results}

The size of catchment areas varied from 300000 to 1 million. Four services classified themselves as operating nationwide.

Services varied considerably in the number of staff employed. On average, they employed 1.2 full-time and 5.4 part-time staff. However, there is considerable variance as indicated by standard deviations of 1.8 for fulltime and 4.2 for part-time staff.

With respect to the professional background of staff, 15 services had one or more clinical psychologist and 12 had a least one psychiatrist in the team. All teams had either a clinical psychologist or a psychiatrist or both. 
Box 1. Information collected by the survey of UK traumatic stress services

Profile of professionals: psychiatrists, clinical psychologists, counsellors, occupational therapists, social workers, nurses, bicultural workers

Type of referrals: self-referral, primary, secondary, tertiary services or other

Number of patients referred and seen in a year prior to carrying out a survey: number of referred and number of seen patients; average waiting time for the first appointment

Treatment sessions: waiting time, separate assessment and treatment session, average number of sessions and range of sessions of complete treatment

Refugees: percentage of overall referrals, most frequent countries of origin, interpreting services needed

Patients' age-groups: under 18, 18-40, 40-65, over 65

Treatment methods: psycho-education, revisiting/reexposure, behavioural techniques, cognitive techniques, psychodynamic techniques, relaxation, EMDR

Support: medico-legal, social support, vocational training

Type of therapy: individual, group, couple and family therapy

Type of supervision: peer, internal, external

Type of outcome measures for treatment effectiveness

EMDR, eye movement desensitisation and reprocessing.

Seven services employed nurses, five a counsellor, four services a social worker, two a bicultural therapist and none employed an occupational therapist.

Under the category of 'other' personnel, staff included speech and language therapists, behavioural therapists, clinical psychology trainees, art therapists and a teacher.

Referral pathways included five services that accept self-referrals, 13 services from primary care, and 15 from secondary or tertiary care.

The numbers of patients seen in the year prior to the survey varied from 17 to 200 . Four services had fewer than 50 patients, five between 50 and 100, five between 100 and 150 and two services had more than 150 patients (data for one service are missing). New referrals in the previous year varied between 25 and 260 in these services. Yet, a significant percentage seems to have been seen as inappropriate and, therefore, not seen. However, the survey did not ascertain how many were inappropriate or why.

Waiting times were between 2 and 28 weeks and were on average 11 weeks. All but one service had separate assessment and treatment sessions. The mean number of treatment sessions is 12 (s.d. $=5$ ). However, most centres indicated that the number of sessions could vary significantly, and that they do not have exact figures on how many sessions over what period of time are provided on average.

In four services, fewer than $10 \%$ of referrals were refugees. In seven services the proportion was between
10 and $50 \%$ and in six services more than $50 \%$. The most common countries of origin were Iraq, former Yugoslavia, Iran, Afghanistan, Sri Lanka, Sierra Leone, Somalia and Turkey.

Most services were designated for adults aged between 18 and 65 years. Only one service provided for children (95\% of patients under 18$)$, and two services had more than $5 \%$ of patients over 65 years.

In terms of therapeutic methods, 16 services provided cognitive-behavioural techniques the components of which included relaxation and breathing controlled techniques, cognitive restructuring, psychoeducation about trauma, in vivo exposure to feared stimuli and imaginal exposure to traumatic memories. In addition, 13 services reported the use of eye movement desensitisation and reprocessing (EMDR). Only four services offered psychodynamic techniques.

All services provided individual psychotherapy. Six also provided group therapy, and eight couple or family therapy. The model preferred for each of these treatments was not specified.

All services provided internal supervision which includes peer supervision. Four services had regular external supervision.

Three services provided social support and one service vocational training. Eleven services had a policy to provide medico-legal reports for their patients.

Fifteen of the 17 services routinely measured treatment outcome. Every centre had different protocols. Four centres used structured clinical interviews to diagnose PTSD: Clinician Administered PTSD Scale (CAPS; Blake et al, 1996); Structured Clinical Interview for DSM-IV Disorders (SCID; First et al, 2002). Self-report measures for PTSD including the Impact of Event Scale (IES/IES-R; Horowitz et al, 1979; Weiss \& Marmar, 1997) were used by 12 centres and the Posttraumatic Diagnostic Scale (Foa et $\mathrm{al}, 1997)$ by six centres. Mood questionnaires included: Beck Depression Inventory (BDI/BDI-II; Beck \& Stear, 1987; Beck et al, 1988) used by 11 centres; Beck Anxiety Inventory (BIA; Beck \& Stear, 1988) by five centres; Hamilton Depression Rating Scale (HRSD; Hamilton, 1967) by two centres; General Health Questionnaire (GHQ; Goldberg \& Hillier, 1979); Core Assessment Outcome Package for the Care Programme Approach (CORE; Evans et al, 2000) by four centres each. In addition, three centres used different types of quality of life scale.

Attempts to cluster centres based on the percentage of refugee referrals, referral pathways, number of patients seen and other variables as assessed in this survey did not reveal any meaningful grouping, and thus further statistical comparison between centres was not conducted.

Finally, we tested the association between number of staff (using an estimate of 0.5 full-time for each parttime staff member). Non-parametric Spearman correlations revealed a significant positive association between the number of patients seen and the number of psychiatrists in the team $\left(r_{s}=0.659, P<0.01\right)$. However, neither the number of clinical psychologists $\left(r_{s}=-0.292\right.$, $P>0.05)$ nor the total number of staff $\left(r_{s}=0.197\right.$, $P>0.05)$ was significantly linked with the number of patients seen. original papers 


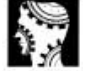

original

papers

\section{Discussion}

The survey shows that most specialised trauma services across the UK have several common features, most prominently fairly short therapeutic programmes involving cognitive-behavioural techniques, including psychoeducation and EMDR. These methods have been shown to be effective in the treatment of PTSD (Sherman, 1998; Van Etten \& Taylor, 1998) and are recommended by the NICE guidelines.

The range of professionals employed in the teams varies and might reflect the importance of multidisciplinary working in the treatment of those with post-traumatic stress syndrome. Yet, it is surprising that some teams work without either a psychiatrist or a clinical psychologist, whose expertise would seem to be essential for such services.

Most services routinely assess outcome, although they do not necessarily use the same instruments. Although local ownership and specific interests may be good reasons for using different measures, a uniform protocol with at least one or two instruments used by all centres would clearly be an advantage for evaluation of treatment outcome. For instance, a protocol could be the basis to establish a common UK-wide database on outcome of specialised treatment, and facilitate a reliable comparison between different service organisations and programmes. Thus, it might be possible to benchmark expected outcomes and identify influential factors. For example, it would be interesting to see whether a population with a higher proportion of refugees is likely to have a less favourable outcome or not, and to what extent this depends on features of the provided programme. What a uniform protocol cannot overcome, however, are the difficulties in obtaining complete data-sets of outcome assessments in routine treatment of an often mobile and challenging clientele (d'Ardenne et al, 2005).

Most centres provide medico-legal reports in support of their clients, which is a controversial issue. The potential or actual provision of such reports can arguably impact on the therapeutic alliance between client and clinician and change treatment outcome. Collaboration between centres should be considered as a means of preventing clinicians from taking an incompatible role of therapist and impartial expert. It should be noted that this refers to reports by 'professional witnesses', i.e. reports by clinicians concerning patients from the same service. We did not ask about 'independent expert' work for other patients outside the trauma centres.

Teams that have larger numbers of psychiatrists tend to see more patients. However, there was no significant association between a greater number of total staff and number of patients seen. Although this may have been partly because of the small sample size and the fact that some of the staff sessions were used for teaching rather than therapeutic work, this negative finding suggests that some services might increase efficiency, in terms of seeing more patients with the same resources, through organisational change. Alternatively, smaller services may be more flexible or have other advantages that enable them to see more patients with fewer resources.

In conclusion, the recent publication of the NICE guidelines on treatment of PTSD (National Collaborating
Centre for Mental Health, 2005) will prompt services to comply with evidence-based practice. Specialised services already appear to employ such methods, but may have to develop even more harmonised practice according to the guidelines. However, the guidelines do not cover the organisation of specialised services and the routine measurement of outcome. The survey shows that services can improve on both aspects, and stronger collaboration will probably be helpful.

\section{Acknowledgement}

The authors thank members of the UK Trauma Group for their participation in the survey.

\section{References}

BECK, A. \& STEER, R. A. (1987) Manual for the Revised Beck Depression Inventory. San Antonio, TX Psychological Corporation.

BECK, A. \& STEER, R. A. (1988) Manual for the Beck Anxiety Inventory. San Antonio,TX: Psychological Corporation.

BECK, A.T., STEER, R. A. \& GARBIN

M. G. (1988) Psychometric propoerties of the Beck Depression Inventory:

Twenty-five years of evaluation. Clinical Psychology Review, 8, 77-100.

BERTHOLD, S. M. (2000) War traumas and community violence:

psychological, behavioral and academic outcomes among Khmer refugee adolescents. Journal of Multicultural Social Work, 8, 15-46.

BLAKE, D. D. WEATHERS, F.W., NAGY, L. M., et al (1996) ClinicianAdministered PTSD Scale for DSM-IV. Current and Lifetime Diagnostic Version. Boston: National Center for Posttraumatic Stress Disorder. d'ARDENNE, P., CAPUZZO, N., FAKHOURY, F., et al (2005) Subjective quality of life and posttraumatic stress disorder. Journal of Nervous and Mental Disease, 193, 62-65.

EVANS, C., MELLOR-CLARK, J., MARGISON, F., et al (2000) CORE: Clinical Outcomes in Routine Evaluation Journal of Mental Health, 9, 247-255.

FIRST, M. B., SPITZER, R. L., GIBBON, M., et al (2002) Structured Clinical Interview for DSM-IV-TR Axis I Disorders. New York: Biometrics Research.

FOA, E. B., CASHMAN, L., JAYCOX, L., et al (1997) The validation of a selfreport measure of posttraumatic stress disorder: The Posttraumatic Diagnostic Scale. Psychological Assessment, 9 445-451.

GOLDBERG, D. \& HILLIER, V. F. (1979) A scaled version of the General Health

*Jelena Jankovic Gavrilovic Academic Unit, Newham Centre for Mental Health, London E13 8SP, and Unit for Social and Community Psychiatry, University of London, e-mail: j.jankovic@qmul.ac.uk, Patricia d'Ardenne Institute of Psychotrauma, East London and the City Mental Health NHS Trust,

Maria Bogic Unit for Social and Community Psychiatry, University of

London, Nicoletta Capuzzo Institute of Psychotrauma, East London and the City Mental Health NHS Trust, Stefan Priebe Unit for Social and Community Psychiatry, University of London and Institute of Psychotrauma, East London and the City Mental Health NHS Trust 